\title{
JOGOS VETORIAIS DIFUSOS COMO FERRAMENTA DE ANÁLISE MULTICRITÉRIO NA AVALIAÇÃO DE EMPRESAS
}

\author{
Adriana Kroenke \\ Universidade Federal do Paraná - UFPR \\ Universidade Regional de Blumenau - FURB \\ Rua Antônio da Veiga, 140 - Blumenau-SC \\ didlen@terra.com.br \\ Volmir Eugênio Wilhelm \\ Universidade Federal do Paraná - UFPR \\ Jardim da Américas, 980 - Curitiba - PR \\ volmirw@gmail.com
}

\begin{abstract}
RESUMO
O posicionamento contábil de empresas é tema recorrente em ambientes de investimento. Esta pesquisa tem por objetivo avaliar o posicionamento contábil das empresas do setor de metalurgia e siderurgia listadas na BM\&FBovespa por meio da teoria dos jogos multicriteriais. São usados quatro lotes de indicadores econômico-financeiros. O primeiro formado por indicadores de liquidez: liquidez geral (LG), liquidez corrente (LC) e liquidez seca (LS). O segundo lote composto por indicadores de endividamento: imobilização do patrimônio líquido (IPL), participação de capital de terceiros (PCT) e composição do endividamento (CE). O terceiro grupo formado por indicadores de rentabilidade: margem líquida (ML), retorno sobre o ativo (ROA), e retorno sobre o patrimônio líquido (ROE). Por último, mas não menos importante usou-se os indicadores de atividade: prazo médio de estoques (PME), prazo médio de fornecedores (PMF) e prazo médio de recebimento (PMR). A leitura é feita usando as empresas como estratégias do jogador I e os indicadores econômico-financeiros como sendo as estratégias do jogador II. O ranking foi inspirado nos trabalhos de Nishizaki e Sakawa (2001), usando metas difusas. O posicionamento contábil obtido reflete os resultados que são percebidos em análise pormenorizada dos dados sob ótica contábil.
\end{abstract}

Palavras-chave: Teoria dos jogos. Jogos multicriteriais. Metas difusas.

\begin{abstract}
The placement of accounting firms is a recurring theme in investment environments. This research aims to evaluate the placement of accounting companies in the iron and steel listed on the BM\&FBovespa by means of multicriteria game. Use four lots of financial indicators. The first consists of liquidity indicators: overall liquidity (LG), current ratio (LC) and liquidity dries (LS). The second batch consists of debt indicators: Immobilization of equity (IPL), share of debt (PCT) and debt composition (CE). The third group of indicators of profitability: net margin (ML), return on assets (ROA) and return on equity (ROE). Last but not least was used activity indicators: average period of inventory (PME), midterm suppliers (PMF) and average collection period (PMR). Reading is done using business strategies as player I and financial indicators as the strategies of player II. The ranking was inspired by the work of Sakawa and Nishizaki (2001), using diffuse goals. The positioning accounting reflects the results obtained are perceived in detailed analysis of the data under accounting perspective.
\end{abstract}

Keywords: Game theory. Multicriteria game. Diffuse goals. 
A teoria dos jogos proporciona modelos de situações reais, pelos quais frequentemente, as conclusões que estes modelos fornecem são somente pautas gerais de comportamento, que proporcionam normas de atuação mais precisas tanto quanto o modelo reflita com mais perfeição a realidade. Tudo isso fez com que a teoria dos jogos crescesse dentro da Pesquisa Operacional, demonstrando possuir suficiente interesse e aplicabilidade para ser estudada como disciplina independente (DIMAND, 1996).

Dentro das organizações, quando se lida com problemas decisórios é certo que serão enfrentadas situações de conflito de interesse (NISHIZAKI e SAKAWA, 2001), competição e cooperação parcial. Estas características são consideradas a essência dos problemas decisórios.

A teoria dos jogos é usada como uma ferramenta analítica poderosa na solução de problemas decisórios ou sistemas competitivos. Exemplos clássicos variados são encontrados nos trabalhos de Neumann e Morgenstern (1944), Harsanyi (1977), Harsanyi e Selten (1988), Fudenberg e Tirole (1991) e Owen (1995).

Os resultados da análise e resolução de problemas de tomada de decisão nem sempre são apropriadas e adequadas aos problemas da vida real caso os parâmetros dos modelos matemáticos para a tomada de decisão são determinados sem considerar a incerteza e a imprecisão presentes em sistemas competitivos.

Os sistemas competitivos formam a matéria-prima deste trabalho. Em especial, entre organizações empresariais, que frequentemente enfrentam difíceis decisões devido a necessidade em cobrir vários imperativos, geralmente conflitantes, como por exemplo, preço e qualidade. Caso o preço seja tomado como critério de decisão, o decisor arisca-se a adquirir um bem pouco durável. Caso a qualidade seja o critério de decisão, então é bem possível que o valor a ser desembolsado seja um valor mais caro. Segundo Barba-Romero e Pomerol (1997, p. 5) "quando os desejos entram em conflito, a decisão resultará de um compromisso".

Quando dois oponentes elegem suas estratégias (alternativas), não só os resultados apresentam uma soma não-nula, mas ela também possui uma forma de um vetor ao invés de um escalar. Jogos reais entre dois personagens, devem ser entendidos como sequências de ganhos e perdas, em que não necessariamente o ganho de um é a perda do outro em cada movimento. Os jogadores não são imediatamente ganhadores ou perdedores. $\mathrm{O}$ uso de estratégias mistas ou randômicas nem sempre são adequadas e a cooperação muitas vezes substitui a concorrência.

A leitura de cada empresa segundo seus vetores formados por índices econômicofinanceiros serão o escopo desta pesquisa. Estes vetores serão obtidos das demonstrações contábeis, que nesta investigação serão os indicadores de liquidez, endividamento, rentabilidade e atividade das empresas de metalurgia e siderurgia listadas na BM\&FBovespa. A partir desses indicadores é possível estabelecer o posicionamento contábil dentro do seu setor, tomadas aqui como sendo suas concorrentes. Este posicionamento surge na forma de um ranking, havendo uma série de autores que já realizaram investigações com cestas compostas por diversos indicadores por meio de métodos que compõe a família de técnicas de análise decisória multicriterial, como são os trabalhos de Kroenke (2009), Rocha (2010), Rodrigues Jr. (2011), Krespi (2012), Kreuzberg (2013) e Kaveski (2013).

\section{JOGOS DIFUSOS BIPESSOAIS DE SOMA ZERO}

A análise segue o esquema introduzido por Zeleny (1982) para jogos com múltiplos pagamentos. Zeleny propôs um vetor aos coeficientes, variando parâmetros na análise dos jogos. Cook (1976) introduziu um vetor de metas (goal vector) e abordou esses jogos na forma de problemas de programação por metas.

A abordagem difusa considerada nessa pesquisa toma em conta a ambiguidade de julgamento, expressada na forma de metas difusas. Assume-se que cada jogador (I e II) possui 
metas difusas, não claras, que podem ser interpretadas por meio de graus de satisfação no retorno dos pagamentos.

Com efeito, a tomada de decisão não envolve apenas ambiguidade, mas também a imprecisão das informações. Quando um sistema competitivo é modelado como sendo um jogo de soma-zero bipessoal, os elementos da matriz de pagamentos são avaliados, utilizando informações disponíveis nos sistemas competitivos, no entanto, já que a informação está disponível, nem sempre é preciso. Assim, os elementos da matriz de pagamentos podem ser tomados como números difusos (DUBOIS e PRADE, 1980) a fim de expressar a imprecisão na informação (SAKAWA e NISHIZAKI, 1994).

Um jogo bipessoal de soma-zero com objetivos difusos difere de jogos convencionais em dois pontos. Primeiro, cada jogador tem um objetivo difuso para um retorno. Por exemplo, uma meta na gestão de vendas define uma empresa privada, enquanto uma empresa pública pode fixar um conjunto de metas de infraestrutura. Uma meta para um objetivo é caracterizado por um valor (um ponto). A diferença entre o valor do objetivo e um valor de realização pode ser interpretado como uma sub-realização ou uma sobre-realização, que os tomadores de decisão (jogadores) tentam minimizar. Por outro lado, um objetivo distorcido é caracterizado por uma função de pertinência, mapeando um domínio de retornos em graus de realização do objetivo distorcido, ou seja, no intervalo [0,1], no qual um jogador tenta maximizar o seu grau de realização para o objetivo difuso. Esses objetivos distorcidos podem ser interpretados como o grau de satisfação a uma recompensa.

Em segundo lugar, vários retornos são introduzidos nos jogos, o que leva a decisões com múltiplos objetivos. Além disso, podemos conectar cada um dos objetivos do problema com cada um dos retornos do jogo.

A pesquisa pretende acomodar a natureza imprecisa do julgamento humano, assumindo que cada jogador tem um objetivo difuso para cada objetivo claro e o conceito de solução consiste aqui na maximização do grau de consecução do objetivo difuso. Sakawa e Nishizaki (1994) afirmam que a solução maximin com respeito ao grau de realização de um objetivo difuso pode ser definido na forma de um problema de programação matemática, que para o cálculo da solução maximin pode ser reduzido a um problema de programação linear, quando cada função de pertinência é identificada como uma função linear ou uma função linear por partes. Particularmente, quando funções de pertinência de ambos os jogadores são simétricas e lineares em um jogo de único objetivo, já está provado que a propriedade da solução de equilíbrio se mantém (COOK, 1976).

Campos (1989) explorou jogos bipessoais de soma-zero com ganhos difusos. O problema tratado foi um jogo de um único objetivo, formulando a situação modelo minimax na forma de um problema de programação matemática difuso. Sakawa e Nishizaki (1994) consideraram a situação para jogos bipessoais multiobjetivos de soma-zero com ganhos difusos e objetivos difusos. Nesta pesquisa será abordado apenas a situação dos objetivos difusos.

\section{NÚMEROS DIFUSOS}

Os conjuntos difusos são usados para modelar informação vaga (KAUFMANN e GUPTA, 1985). De maneira simplista, a noção de conjunto difuso pode ser abordada como uma generalização da noção clássica, costumeiramente denominados conjuntos crisp, que objetiva representar conjuntos cujas fronteiras não são claras.

Quando da definição de um conjunto, sua função característica pode ser generalizada de maneira a associar a cada elemento do conjunto universo um valor, em determinado intervalo (geralmente $[0,1]$ ), que reflete o grau de pertinência do elemento ao conjunto sendo definido. Tal função é chamada de função de pertinência e o conjunto definido por ela é denominado conjunto difuso (HEIN e DADAM, 2009).

Esta pesquisa não necessita de um aprofundamento no tema, contudo trabalhará com números difusos. Um número difuso segundo Kandel (1986) “é um subconjunto dos números 
reais, convexo e normal". Pode-se definir um número difuso (fuzzy number) em qualquer conjunto referencial totalmente ordenado, como é o caso dos reais, contudo pode ser usado os $\mathbb{R}^{+}, \mathbb{Z}$ e $\mathbb{N}$, por exemplo. Nos casos em que a função de pertinência é uma função contínua diz-se que o número difuso é contínuo.

\section{JOGOS MULTICRITERIAIS COM METAS DIFUSAS}

Considere-se o seguinte jogo vetorial, portanto multiobjetivo, bipessoal de soma-zero, representado pelas matrizes de pagamento:

$$
A^{1}=\left(\begin{array}{ccc}
a_{11}^{1} & \cdots & a_{1 n}^{1} \\
\vdots & \ddots & \vdots \\
a_{m 1}^{1} & \cdots & a_{m n}^{1}
\end{array}\right), \ldots, A^{r}=\left(\begin{array}{ccc}
a_{11}^{r} & \cdots & a_{1 n}^{r} \\
\vdots & \ddots & \vdots \\
a_{m 1}^{r} & \cdots & a_{m n}^{r}
\end{array}\right)
$$

Assumindo que cada um dos jogadores tenham $r$ objetivos. As estratégias puras correspondem as linhas e colunas de cada matriz $A^{k}{ }_{3}, k=1, \ldots, r$ para o jogador I e jogador II, respectivamente. Nomeadamente, quando o jogador I elege uma estratégia pura $i \in I=\{1, \ldots, n\}$ e o jogador II elege a estratégia pura $j \in J=\{1, \ldots, m\}$ recebendo o jogador I o vetor de pagamentos $\left(a_{i j}^{1}, a_{i j}^{2}, \ldots, a_{i j}^{r}\right)$ do jogador II.

Seja $x \in X=\left\{x \in \mathbb{R}^{n} / \sum_{i=1}^{m} x_{i}=1 ; x_{i} \geq 0 ; i=1, \ldots, m\right\}$ uma estratégia mista para 0 jogador I e seja $y \in Y=\left\{y \in \mathbb{R}^{n} / \sum_{j=1}^{n} y_{j}=1 ; y_{j} \geq 0 ; j=1, \ldots, n\right\}$ uma estratégia mista do jogador II. Este trabalho assume que um jogador possui uma meta difusa (fuzzy goal) para cada um dos objetivos, que expressa um grau de satisfação por um pagamento (payoff).

Definição 1: (Fuzzy Goal) Seja o domínio do k-ésimo pagamento para o jogador I dado por $D^{k} \in \mathbb{R}$, então a meta difusa $\mu^{k}$ com respeito ao k-ésimo pagamento para o jogador I é o conjunto difuso $D^{k}$ caracterizado pela função de pertinência:

$$
\mu^{k}: D^{k} \rightarrow[0,1]
$$

Assume-se que o jogador I especifica um valor finito $\underline{a}$ de pagamento para qual o seu grau de satisfação é nulo e um valor finito $\bar{a}$ de pagamento para o qual seu grau de satisfação é 1. Para um valor indesejado $\mathrm{p}$, menor que $\underline{a}$ fica definido que $\mu^{k}(p)=0$, para um valor desejado $\mathrm{p}$, maior que $\bar{a}$ fica estabelecido que $\mu^{k}(p)=1$, e para $\underline{a} \leq p \leq \bar{a}, \mu^{k}(p)$ é contínuo estritamente crescente.

A função de pertinência para uma meta difusa pode ser interpretada como um grau de atendimento a um pagamento. Caso o jogador tenha dois pagamentos distintos, ele irá eleger o pagamento que possua o maior grau de pertinência em relação ao outro. Este procedimento levará a maximização do grau de atendimento da meta difusa.

Assume-se que o jogador I suponha que o jogador II escolha a estratégia y que minimiza o grau de atendimento as metas difusas $\mu^{k}(x, y)$ do jogador I. De similar modo o jogador II usa a mesma linha de raciocínio para o jogador I. Assim, o grau de atendimento as metas difusas, é assumindo que ele escolha a estratégia $x$, em que $v^{k}(x)=\min _{y \in Y} \mu^{k}(x, y)$.

Assim, o jogador I escolhe a estratégia que maximiza seu grau de atendimento as metas difusas $v^{k}(x)$. Em resumo, assume-se que o jogador atua de acordo com o princípio maximin em termos de graus de atendimento aos seus objetivos (metas) difusas. 
Tudo isso pode ser também interpretado como sendo um problema de otimização de um vetor com múltiplos objetivos, ou seja, na forma de um jogo vetorial difuso. Entretanto, para cada uma das unidades de medida para os objetivos, estas podem ser transformadas em um grau de atendimento da meta difusa como uma nova unidade de medida, considerando problemas maximin em termos da maximização do grau de atendimento a meta difusa.

Definição 2: (Solução maximin com respeito ao grau de atendimento da meta difusa). Seja a função de pertinência de agregação da meta difusa do jogador I dada por $\mu(x, y)$ quando os jogadores I e II elegem as estratégias $x$ e $y$, respectivamente. Então o valor maximin do jogador I com respeito ao grau de atendimento da meta difusa é $\max _{x \in X} \min _{y \in Y} \mu(x, y)$.

De similar modo, para o jogador II o valor minimax com respeito ao grau de atendimento a meta difusa é $\min _{y \in Y} \max _{x \in X} \bar{\mu}(x, y)$, onde $\bar{\mu}(x, y)$ é a função de pertinência do jogador II.

A solução maximin pode ser considerada como sendo a maximização da função a qual é o valor minimal da função com respeito as variáveis de decisão do oponente.

A operacionalização disto é feita tomando a função de pertinência da meta difusa do jogador I para o k-ésimo objetivo como sendo $\mu^{k}\left(x A^{k} y\right)$ para todo par de estratégias mistas $(x, y)$.

Assumindo como a função de pertinência $\mu^{k}\left(x A^{k} y\right)$ para a meta difusa como sendo linear, ela é representada por:

$$
\mu^{k}\left(x A^{k} y\right)=\left\{\begin{array}{cl}
0 & \text {;se } x A^{k} y \leq \underline{a}^{k} \\
1-\frac{\bar{a}^{k}-x A^{k} y}{\bar{a}^{k}-\underline{a}^{k}} & ; \text { se } \underline{a}^{k} \leq x A^{k} y \leq \bar{a}^{k} \\
1 & \text {;se } \bar{a}^{k} \leq x A^{k} y
\end{array}\right.
$$

Onde $\underline{a}^{k}$ é o pagamento que retorna com o pior nível de satisfação do jogador I com respeito ao k-ésimo objetivo e $\bar{a}^{k}$ é o pagamento dado ao melhor grau de satisfação para o jogador I, com respeito ao mesmo k-ésimo objetivo.

$$
\begin{aligned}
& \underline{a}^{k}=x^{\circ} A^{k} y^{\circ}=\min _{x \in X} \min _{y \in Y} x A^{k} y=\min _{i \in I} \min _{j \in I} a_{i j}^{k} \\
& \bar{a}^{k}=x^{1} A^{k} y^{1}=\max _{x \in X} \max _{y \in Y} x A^{k} y=\max _{i \in I} \max _{j \in J} a_{i j}^{k}
\end{aligned}
$$

Empregando a regra de decisão difusa de Bellman e Zadeh (1970), que muitas vezes é usada em problemas decisórios em cenários difusos, chega-se a regra de agregação de múltiplas metas difusas. Expressando como a função de pertinência de agregação de metas difusas por:

$$
\mu(x, y)=\min _{k \in K} \mu^{k}\left(x A^{k} y\right)
$$

Tomando cada uma das funções de perinência como lineares, a função de pertinência de agregação de metas difusas é expressada como:

$$
\mu(x, y)=\min _{k \in K}\left[1-\frac{\bar{a}^{k}-x A^{k} y}{\bar{a}^{k}-\underline{a}^{k}}\right]
$$




$$
\begin{gathered}
\mu(x, y)=\min _{k \in K}\left[\sum_{i=1}^{m} \sum_{j=1}^{n} \frac{a_{i j}^{k}}{\bar{a}^{k}-\underline{a}^{k}} x_{i} y_{j}-\frac{\underline{a}^{k}}{\bar{a}^{k}-\underline{a}^{k}}\right] \\
\mu(x, y)=\min _{k \in K}\left[\sum_{i=1}^{m} \sum_{j=1}^{n} \hat{a}_{i j}^{k} x_{i} y_{j}+c^{k}\right]
\end{gathered}
$$

Onde: $\hat{a}_{i j}^{k}=\frac{a_{i j}^{k}}{\bar{a}^{k}-\underline{a}^{k}}$ e $c^{k}=-\frac{a^{k}}{\bar{a}^{k}-\underline{a}^{k}}$

Toda esta análise pode ser transcrita em um problema de programação linear, como Nishizaki e Sakawa (2001) descrevem no teorema.

Teorema 1: Para jogos multiobjetivos bipessoais de soma-zero com funções de pertinência de metas difusas dadas em sua forma linear, agregadas pela regra de decisão difusa (Bellman-Zadeh), a solução maximin para o jogador I, com respeito ao grau de atendimento a meta difusa agregada é dada pelo problema de programação linear.

\section{$\operatorname{Max} \lambda$}

$$
\begin{gathered}
\text { Sujeito } a: \hat{a}_{1 j}^{1} x_{1}+\cdots+\hat{a}_{m j} x_{m}+c^{1} \geq \lambda ; \mathrm{j}=1, \ldots, \mathrm{n} \\
\cdots \\
\qquad \begin{array}{c}
\hat{a}_{1 j}^{r} x_{1}+\cdots+\hat{a}_{m j}^{r} x_{m}+c^{1} \geq \lambda ; \mathrm{j}=1, \ldots, \mathrm{n} \\
x_{1}+\cdots+x_{m}=1 \\
x_{i} \geq 0, i=1, \ldots, m
\end{array}
\end{gathered}
$$

Demonstração:

Usando a regra de decisão difusa (Bellman - Zadeh) como regra de agregação das metas difusas do jogo multiobjetivo bipessoal de soma-zero, tem-se a formação de um problema maximin:

$$
\max _{x \in X} \min _{y \in Y} \mu(x, y)=\max _{x \in X} \min _{y \in Y} \min _{k \in K} \mu^{k}\left(x A^{k} y\right)
$$

Que pode ser reescrito:

$$
\max _{x \in X} \min _{y \in Y} \mu(x, y)=\max _{x \in X} \min _{y \in Y} \min _{k \in K}\left[\sum_{i=1}^{m} \sum_{j=1}^{n} \hat{a}_{i j}^{k} x_{i} y_{j}+c^{k}\right]
$$

Introduzindo uma variável auxiliar dada pelo vetor

$$
\begin{aligned}
& z=\left(z_{1}, \ldots, z_{r}\right) \in Z=\left\{z \in \mathbb{R}^{r} / \sum_{k=1}^{r} z_{k}=1 ; z_{k} \geq 0 ; k=1, \ldots, r\right\}, \text { tem-se: } \\
& \max _{x \in X} \min _{y \in Y} \mu(x, y)=\max _{x \in X} \min _{y \in Y} \min _{z \in Z}\left[\sum_{i=1}^{m} \sum_{j=1}^{n} \sum_{k=1}^{r} \hat{a}_{i j}^{k} x_{i} y_{j} z_{k}+\sum_{k=1}^{r} c^{k} z_{k}\right]= \\
& =\max _{x \in X} \min _{y \in Y} \min _{z \in Z}\left[\sum_{i=1}^{m} \sum_{j=1}^{n} \sum_{k=1}^{r} \hat{a}_{i j}^{k} x_{i} y_{j} z_{k}+\sum_{j=1}^{n} y_{i} \sum_{k=1}^{r} c^{k} z_{k}\right]=
\end{aligned}
$$




$$
\begin{aligned}
& =\max _{x \in X} \min _{y \in Y} \min _{z \in Z}\left[\sum_{i=1}^{m} \sum_{j=1}^{n} \sum_{k=1}^{r} \hat{a}_{i j}^{k} x_{i} y_{j} z_{k}+\sum_{j=1}^{n} \sum_{k=1}^{r} c^{k} y_{j} z_{k}\right]= \\
& =\max _{x \in X} \min _{y \in Y} \min _{z \in Z} \sum_{j=1}^{n} \sum_{k=1}^{r}\left[\sum_{i=1}^{m} \hat{a}_{i j} x_{i} y_{i} z_{k}+c^{k} y_{j} z_{k}\right]
\end{aligned}
$$

Fazendo a transformação:

$w=\left(w_{1}, \ldots, w_{n r}\right)=\left(y_{1} z_{1}, \ldots, y_{n} z_{r}\right)$

Em que $w \in W=\left\{w \in \mathbb{R}^{n r} / \sum_{l=1}^{n r} w_{l}=1 ; w_{l} \geq 0 ; l=1, \ldots, n r\right\}$ com a qual a expressão $\max _{x \in X} \min _{y \in Y} \mu(x, y)$ pode ser reduzida.

$$
\begin{aligned}
& \max _{x \in X} \min _{y \in Y} \mu(x, y)=\max _{x \in X} \min _{w \in W} \sum_{l=1}^{n r}\left[\sum_{i=1}^{m} \hat{a}_{i j}^{k} x_{i} w_{i}+c^{k} w_{l}\right]= \\
& =\max _{x \in X} \min _{w \in W} \sum_{i=1}^{n r}\left[\sum_{i=1}^{m} \hat{a}_{i j}^{k} x_{i}+c^{k}\right] w_{l}
\end{aligned}
$$

Com isso é possível determinar a estratégia $x^{*}$ resolvendo o problema de programação linear do enunciado.

Para o jogador II, a estratégia minimax é obtida pelo problema de programação linear dual, assim expressado:

\section{$\operatorname{Min} \lambda$}

$$
\begin{gathered}
\text { Sujeito } a: \hat{a}_{i 1}^{1} y_{1}+\cdots+\hat{a}_{i n}^{1} y_{n}+c^{1} \leq \lambda ; \mathrm{i}=1, \ldots, \mathrm{m} \\
\ldots \\
\hat{a}_{i 1}^{r} y_{1}+\cdots+\hat{a}_{i n} y_{n}+c^{r} \leq \lambda ; \mathrm{i}=1, \ldots, \mathrm{m} \\
y_{1}+\cdots+y_{n}=1 \\
y_{j} \geq 0, j=1, \ldots, n
\end{gathered}
$$

A solução do modelo primal e do modelo dual darão o vetor de estratégias, formado por um lado por uma cesta de empresas, tomadas como sendo as estratégias do jogador I e os indicadores contábeis divididos em liquidez, endividamento, rentabilidade e atividade; como sendo as estratégias do jogador II (indicadores). Estes grupos, bem como os indicadores que compõe cada um deles, são definidos a seguir.

\section{MATERIAIS E MÉTODOS}

Nesta pesquisa trata-se de um estudo descritivo que apresenta o ranking das empresas do setor de metalurgia e siderurgia listadas na BM\&FBovespa.

Para atender o objetivo desta investigação faz-se necessário verificar indicadores contábeis apresentados na literatura, o que caracteriza uma pesquisa bibliográfica e o fato de utilizar demonstrações contábeis como fonte de coleta de dados torna esta pesquisa documental, pois, os dados ainda não receberam nenhuma forma de tratamento.

Quanto à abordagem do problema este estudo classifica-se como quantitativo. "O método quantitativo representa, em princípio, a intenção de garantir a precisão dos resultados, evitar distorções de análise e interpretação, possibilitando, consequentemente, uma margem de segurança quanto as inferências" (RICHARDSON, 1989, p. 29). 
A população é definida como o conjunto de elementos que apresentam os atributos necessários para o desenvolvimento do estudo (SILVEIRA, 2004). No caso desta pesquisa, que apresenta os dados dos indicadores de liquidez, endividamento, rentabilidade e atividade. A população, nesta pesquisa, consiste nas 12 empresas de siderurgia e metalurgia listadas na BM\&FBovespa. Todas as empresas apresentaram os dados necessários, logo, nenhuma empresa foi excluída da análise.

A população foi definida intencionalmente, ou seja, consiste em uma população não probabilística e justifica-se pelo acesso às informações contábeis e seu grau de confiabilidade por se tratarem de empresas de capital aberto. As empresas do ramo de siderurgia e metalurgia utilizadas nesta investigação são apresentadas no Quadro 1.

\section{Quadro 1 - Empresas do setor de siderurgia e metalurgia listadas na BM\&FBovespa}

\begin{tabular}{|l|l|l|}
\hline Empresa & Nome do pregão & Atuação \\
\hline Paranapanema & PARANAPANEMA & Artefatos de cobre \\
\hline Fibam Companhia Industrial & FIBAM & Artefatos de Ferro e Aço \\
\hline Mangels Industrial S.A. & MANGELS INDL & Artefatos de Ferro e Aço \\
\hline Metalúrgica Duque S.A. & MET DUQUE & Artefatos de Ferro e Aço \\
\hline Panatlantica S.A. & PANATLANTICA & Artefatos de Ferro e Aço \\
\hline Siderurgica J. L. Aliperti S.A. & ALIPERTI & Artefatos de Ferro e Aço \\
\hline Tekno S.A. - Indústria e Comércio & TEKNO & Artefatos de Ferro e Aço \\
\hline CIA Ferro Ligas da Bahia - FERBASA & FERBASA & Siderurgia \\
\hline CIA Siderurgia Nacional & SID NACIONAL & Siderurgia \\
\hline GERDAU S.A. & GERDAU & Siderurgia \\
\hline Metalurgica Gerdau S.A & GERDAU MET & Siderurgia \\
\hline Usinas SID de Minas Gerais S.A. - USIMINAS & USIMINAS & Siderurgia \\
\hline
\end{tabular}

Fonte: BM\&Fbovespa (www.bm\&fbovespa.com.br).

Os dados foram obtidos das demonstrações contábeis consolidadas, Balanço Patrimonial e Demonstração do Resultado do Exercício por meio do ECONOMÁTICA ${ }^{\odot}$. Foram extraídos os indicadores econômico-financeiros de liquidez, endividamento, rentabilidade e atividade. De cada grupo foram extraídos três indicadores formando um grupo de 12 indicadores analisados: (a) liquidez: liquidez seca, liquidez corrente, liquidez geral, (b) endividamento: imobilização do patrimônio líquido, participação de capital de terceiros, composição do endividamento, (c) rentabilidade: margem líquida, retorno sobre o ativo, retorno sobre o patrimônio líquido, (d) atividade: prazo médio de estoques, prazo médio de fornecedores e prazo médio de recebimento. Estes foram calculados conforme fórmulas extraídas de Matarazzo (2008).

\section{ANÁLISE DE RESULTADOS}

A análise de dados referentes aos indicadores de liquidez, endividamento, rentabilidade e atividade acabam por formar a matriz de pagamentos do jogo multicriterial.

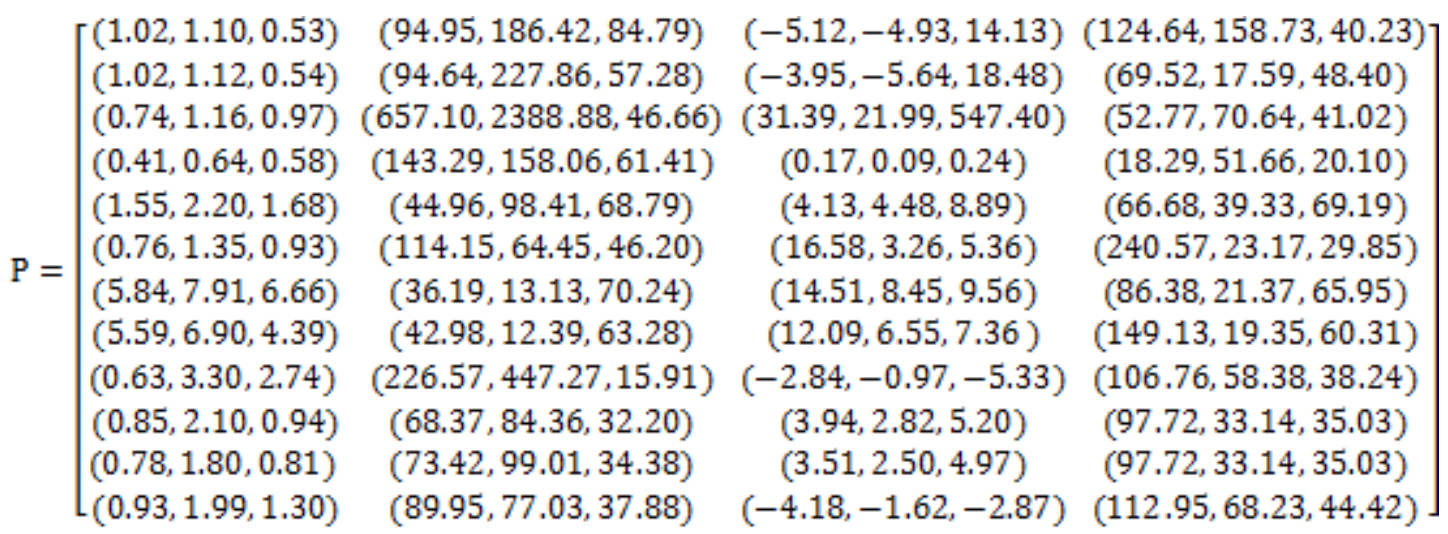


Estes dados são referentes ao exercício 2012. Os valores são adimensionais, ou seja, não possuem uma unidade em especial. Contudo, cada indicador será transformado por meio de uma contração de Lipschitz $d(f(x), f(y)) \leq k d(x, y)$.

Basicamente a contração é feita usando o teorema de Tales, ou seja, em cada um dos 12 indicadores há um máximo $i_{j}^{+} ; j=1, \ldots, 12$ e um mínimo $i_{j}^{-} ; j=1, \ldots, 12$. Fazendo $f\left(i_{j}^{-}\right)=0$ e $f\left(i_{j}^{+}\right)=1, \operatorname{assim} f\left(i_{j}\right)=\frac{i_{j}^{-} i_{\bar{j}}}{i_{j}^{+}-i_{j}}$.

No caso dos indicadores de endividamento pretende-se quanto menor melhor, logo a formulação passa a ser $f\left(i_{j}\right)=1-\frac{i_{j} \bar{i}_{\bar{j}}^{\bar{j}}}{\bar{i}_{j}^{+}-\tilde{i}_{j}}$. Isto também é aplicado ao indicador PME (prazo médio de estoques) e PMR (prazo médio de recebimento).

Assim, a matriz de pagamentos fica definida:

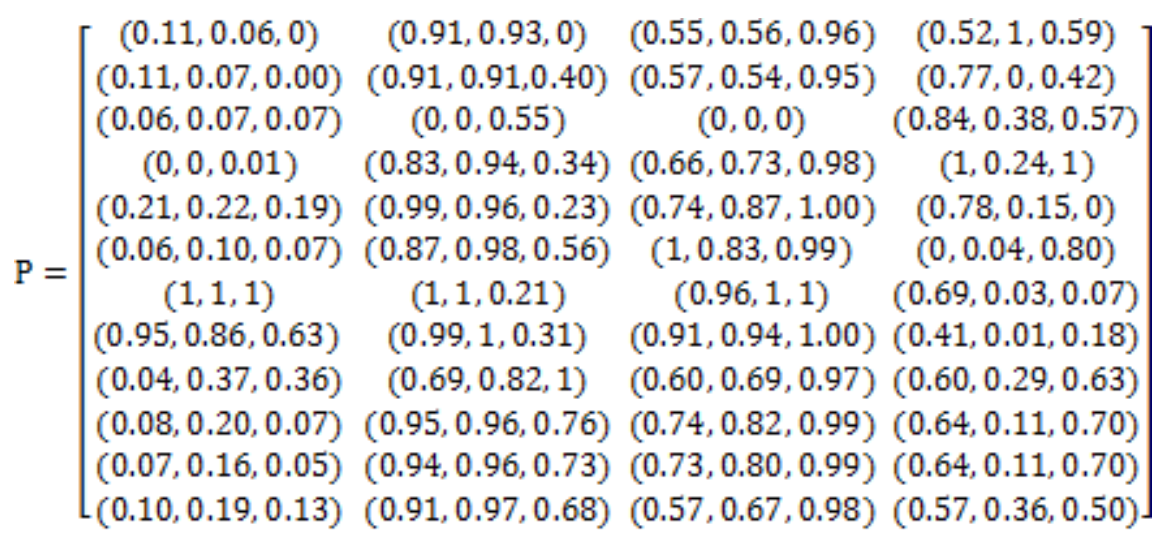

A construção do modelo usa os dados brutos da matriz de pagamentos original P. Isto se justifica no fato da construção aplicada em $\mathrm{P}$ pode ser compreendida como sendo uma préfuzzificação dos dados.

A matriz de pagamentos fuzzificada é criada a partir da análise dividindo cada elemento de $\mathrm{P}$ pela diferença entre o máximo e mínimo de cada grupo de indicadores.

Os valores $c^{k}$ associados a cada grupo de indicadores é dado por:

$c^{1}=-0,054784 \quad c^{2}=-0,005213 \quad c^{3}=0,9706 \quad c^{4}=-0,078901$

Logo o modelo, fica assim construído:

\begin{tabular}{|c|}
\hline $\begin{array}{c}\quad M a x Z=\lambda \\
\quad \text { Sujeito a: } \\
0,136 x_{1}+0,136 x_{2}+0,099 x_{3}+\cdots+0,113 x_{10}+0,104 x_{11}+0,124 x_{12}-0,054784 \geq \lambda \\
0,147 x_{1}+0,149 x_{2}+0,155 x_{3}+\cdots+0,280 x_{10}+0,240 x_{11}+0,266 x_{12}-0,054784 \geq \lambda \\
0,070 x_{1}+0,072 x_{2}+0,130 x_{3}+\cdots+0,126 x_{10}+0,108 x_{11}+0,173 x_{12}-0,054784 \geq \lambda \\
0,040 x_{1}+0,040 x_{2}+0,276 x_{3}+\cdots+0,029 x_{10}+0,031 x_{11}+0,038 x_{12}-0,005213 \geq \lambda \\
0,078 x_{1}+0,096 x_{2}+1,005 x_{3}+\cdots+0,036 x_{10}+0,042 x_{11}+0,032 x_{12}-0,005213 \geq \lambda \\
0,036 x_{1}+0,024 x_{2}+0,020 x_{3}+\cdots+0,014 x_{10}+0,014 x_{11}+0,016 x_{12}-0,005213 \geq \lambda \\
-0,009 x_{1}-0,070 x_{2}-0,056 x_{3}+\cdots+0,007 x_{10}+0,006 x_{11}-0,007 x_{12}+0,9706 \geq \lambda \\
-0,009 x_{1}-0,010 x_{2}-0,039 x_{3}+\cdots+0,005 x_{10}+0,004 x_{11}-0,037 x_{12}+0,9706 \geq \lambda \\
-0,025 x_{1}-0,033 x_{2}-0,971 x_{3}+\cdots+0,009 x_{10}+0,009 x_{11}-0,005 x_{12}+0,9706 \geq \lambda \\
0,559 x_{1}+0,312 x_{2}+0,237 x_{3}+\cdots+0,438 x_{10}+0,438 x_{11}+0,507 x_{12}-0,078901 \geq \lambda \\
0,712 x_{1}+0,079 x_{2}+0,317 x_{3}+\cdots+0,149 x_{10}+0,149 x_{11}+0,306 x_{12}-0,078901 \geq \lambda \\
0,180 x_{1}+0,217 x_{2}+0,184 x_{3}+\cdots+0,157 x_{10}+0,157 x_{11}+0,199 x_{12}-0,078901 \geq \lambda \\
x_{1}+x_{2}+x_{3}+\cdots+x_{10}+x_{11}+x_{12}=1\end{array}$ \\
\hline
\end{tabular}




$$
x_{i} \geq 0 ; i=1, \ldots, 12 ; \lambda \geq 0
$$

Obviamente o modelo precisa ser modificado a ponto de ser introduzido no pacote utilizado (PLM 3.0) por não se encontrar descrito na forma padrão. Após as modificações, sua resolução sequencial levou ao seguinte ranking de posicionamento contábil.

Quadro 2 - Resultados referentes a aplicação do modelo com metas difusas

\begin{tabular}{|c|l|l|c|c|}
\hline POSIÇÃO & \multicolumn{1}{|c|}{ EMPRESA } & \multicolumn{1}{c|}{ VARIÁVEL } & $Z^{*}$ & ESTRATÉGIA \\
\hline $1^{\mathrm{a}}$ & Paranapanema & $x_{1}=0,981$ & 0,03 & Mista \\
\hline $2^{\mathrm{a}}$ & Tekno & $x_{7}=0,947$ & 0,02 & Mista \\
\hline $3^{\mathrm{a}}$ & Panatlântica & $x_{5}=0,962$ & 0,02 & Mista \\
\hline $4^{\mathrm{a}}$ & Ferbasa & $x_{\mathrm{g}}=0,876$ & 0,02 & Mista \\
\hline $5^{\mathrm{a}}$ & Duque & $x_{4}=0,752$ & 0,02 & Mista \\
\hline $6^{\mathrm{a}}$ & Fibam & $x_{2}=0,922$ & 0,018 & Mista \\
\hline $7^{\mathrm{a}}$ & Mangels & $x_{\mathrm{a}}=0,985$ & 0,01 & Pura \\
\hline $8^{\mathrm{a}}$ & Aliperi & $x_{6}=1$ & 0,01 & Pura \\
\hline $9^{\mathrm{a}}$ & Usiminas & $x_{12}=1$ & 0,01 & Pura \\
\hline $10^{\mathrm{a}}$ & Gerdau Met & $x_{11}=1$ & 0,01 & Pura \\
\hline $11^{\mathrm{a}}$ & Gerdau & $x_{10}=1$ & 0,01 & - \\
\hline $12^{\mathrm{a}}$ & Siderúrgica Nacional & $x_{9}=1$ & - & \\
\hline
\end{tabular}

Fonte: Dados da pesquisa.

\section{CONCLUSÕES}

A classificação das empresas por meio de indicadores econômico-financeiros pode sem dúvida ser elaborada por estudiosos da contabilidade, que por meio de seus métodos e técnicas levam a rankings semelhantes aos obtidos pela presente pesquisa. Entretanto, o incremento da cesta de indicadores, da amplicação do horizonte temporal e inclusão de mais empresas no conjunto em investigação fará com que o nível de dificuldade aumente em forma diretamente proporcional, inviabilizando a análise frente as limitações do raciocínio humano dada a complexidade do cenário em estudo. Daí a importância da elaboração de uma metodologia (conjunto de métodos) de auxílio à decisão.

Diante dos resultados desta pesquisa pode-se afirmar que o objetivo foi alcançado. $\mathrm{O}$ modelo consistiu numa formulação mais sofisticada dentre outros modelos de programação linar, apresentando como classificação a empresa Paranapanema em $1^{\mathrm{a}}$ colocação, Tekno $\left(2^{\mathrm{a}}\right)$, Panatlântica $\left(3^{a}\right)$, Ferbasa $\left(4^{a}\right)$, Duque $\left(5^{a}\right)$, Fibam $\left(6^{a}\right)$, Mangels $\left(7^{a}\right)$, Aliperti $\left(8^{a}\right)$, Usiminas $\left(9^{\mathrm{a}}\right)$, Gerdau $\left(10^{\mathrm{a}}\right)$, Gerdau Met $\left(11^{\mathrm{a}}\right)$ e Siderúrgica Nacional $\left(12^{\mathrm{a}}\right)$.

Destaca-se o fato do trabalho ser fortemente inspirado nos trabalhos de Milan Zeleny e por esta pesquisa atestar a possibilidade do uso da programação linear como ferramenta de classificação, em especial em cenários multicriteriais.

\section{REFERÊNCIAS BIBLIOGRÁFICAS}

[1] BARBA-ROMERO, Sergio; POMEROL, Jean-Charles. Decisiones multicriterio: fundamentos teóricos y utilización práctica. Alcalá: Universidada de Alcalá, 1997.

[2] CAMPOS L. Fuzzy linear programming models to solve fuzzy matrix games. Fuzzy Sets and Systems, v. 32, p. 275-289, 1989Segunda referência.

[3] COOK, Wade D. Zero-sum games with multiple goals. Naval Research Logistics Quarterly, v. 23, n. 4, p. 615-621, 1976.

[4] DIMAND, Mary Ann; DIMAND, Robert W. The history of game theory, Volume I: from the beginnings to 1945. London: Routledge, 1996. 
[5] DUBOIS, Didier; PRADE, Henry. Fuzzy sets and systems: theory and applications. New York: Academic Press, 1980.

[6] FUNDENBERG, Drew; TIROLE, Jean. Game theory. Massachusetts: The MIT Press, 1991.

[7] HARSANY, John Charles. Rational behavior and bargaining equilibrium in games and social situations. New York: Cambridge University Press, 1977.

[8] HARSANY, John Charles; SELTEN Reinhard. A general theory of equilibrium selection in games. Massachusetts: The MIT Press, 1988.

[9] HEIN, Nelson; DADAM, Fábio. Teoria Unificada dos Conjuntos. Rio de Janeiro: Editora Ciência Moderna Ltda., 2009.

[10] KANDEL, Abraham. Fuzzy mathematical techniques with applications. New York: Addison-Wesley Publishing Company, 1986.

[11] KAUFMANN, Arnold; GUPTA, M. Introduction to fuzzy arithmetic. New York: Van Nostrad Reinhold Company, 1985.

[12] KAVESKI, Itzhak David Simão. Grau de relacionamento entre os indicadores de Mercado de capitais, os indicadores econômico-financeiros e o retorno da ação nas empresas brasileiras. 2013. 105f. Dissertação (Mestrado em Ciências Contábeis) Programa de Pós-Graduação em Ciências Contábeis da Universidade Regional de Blumenau. Blumenau, 2013.

[13] KRESPI, Nayane Thais. Relação entre os rankings formados pelos indicadores de sustentabilidade e pelos indicadores financeiros tradicionais das empresas candidatas ao ISE: uma aplicação dos métodos displaced ideal e displaced ideal modificado. 2012. 101 f. Dissertação (Mestrado em Ciências Contábeis) - Programa de Pós-Graduação em Ciências Contábeis da Universidade Regional de Blumenau, Blumenau, 2012.

[14] KREUZBERG, Fernanda. Economic indicators versus social indicators: an analysis of companies listed on BM\&FBovespa through the theory of games. 2013. $167 \mathrm{f}$. Dissertation (Master of Accounting) - Program Graduate Accounting, Regional University of Blumenau, Blumenau, 2013.

[15] KROENKE, Adriana. Posicionamento das empresas do setor metal-mecânico listadas na Bovespa: uma aplicação do método AHP. 2009. 110f. Dissertação (Mestrado em Ciências Contábeis) - Programa de Pós-Graduação em Ciências Contábeis da Universidade Regional de Blumenau. Blumenau, 2009.

[16] MATARAZZO, Dante Carmine. Análise financeira de balanços: abordagem gerencial. 7. ed. São Paulo: Atlas, 2008.

[17] NISHIZAKI, Ichiro; SAKAWA, Masatoshi. Fuzzy and multiobjective games for conflict resolution. New York: Physica-Verlag, 2001.

[18] NEUMANN, John von; MORGENSTERN, Oskar. Theory of games and economic behavior. New York: Wiley, 1944.

[19] OWEN, Guillermo. Game theory. San Diego: Academic Press, Third Edition, 1995.

[20] RICHARDSON, Roberto Jarry. Pesquisa social: métodos e técnicas. 2. ed. São Paulo: Atlas, 1989.

[21] ROCHA, Irani. Grau de entropia da informação em indicadores econômico-financeiros das empresas que participam dos níveis de governança corporativa da BM\&FBovespa. 2010. 155f. Dissertação (Mestrado em Ciências Contábeis) - Programa de Pós- 
Graduação em Ciências Contábeis da Universidade Regional de Blumenau. Blumenau, 2010 .

[22] RODRIGUES JUNIOR, Moacir Manoel. Implicações do processo de convergência contábil na análise de desempenho: um estudo por meio da análise envoltória de dados em empresas listadas na BM\&FBovespa. 2012. 170f. Dissertação (Mestrado em Ciências Contábeis) - Programa de Pós-Graduação em Ciências Contábeis da Universidade Regional de Blumenau. Blumenau, 2012.

[23] SILVEIRA, Amélia. (Coord.). Roteiro básico para apresentação e editoração de teses, dissertações e monografias. 2. ed. rev., atual e ampl. Blumenau: Edifurb, 2004.

[24] ZELENY, Milan. Multiple criteria decision making. New Yor: McGraw-Hill, 1982. 